Punjab University Journal of Mathematics (2021),53(9),621-629

https://doi.org/10.52280/pujm.2021.530902

\title{
A Rudimentary Approach to Develop Context for Convexity cum Concavity on Soft Expert Set with Some Generalized Results
}

\author{
Muhammad Ihsan ${ }^{1, *}$, Muhammad Saeed ${ }^{2}$ and Atiqe Ur Rahman ${ }^{3}$ \\ $1,2,3$ Department of Mathematics, \\ University of Management and Technology, Lahore, Pakistan,
}

Email: mihkhb@gmail.com ${ }^{1}$, muhammad.saeed@umt.edu.pk ${ }^{2}$, aurkhb@ gmail.com ${ }^{3}$

Received:12 February, 2021 / Accepted: 03 September, 2021 / Published online: 25, September, 2021

\begin{abstract}
Soft set theory is considered as the preeminent tool to tackle the problems involving vagueness by controlling all complexities of optimization theory, fuzzy set theory and interval theory. Some models have been developed to solve problems in decision making and medical diagnosis with one expert by using this theory. This causes a problem with those who use questionnaires in their research. Soft expert set overcomes this problem and facilitates the user to know the opinion of all experts in one model. The concept of convexity plays a key role to deal optimization, pattern recognition-classification and many other related topics in operation research, numerical analysis and other disciplines of mathematical sciences. In this study, a mathematical cum abstract technique is employed to develop basic concept of convex and concave soft expert sets to deal with their important applications. Some classical results on convexity cum concavity are modified under uncertain multi-decisive environment with the support of explicatory proofs.
\end{abstract}

AMS (MOS) Subject Classification Codes: 26A51, 26B25, 32F17, 35E10, 46N10. Key Words: Soft set, Soft expert set, Convex soft expert set, Concave soft expert set.

\section{INTRODUCTION}

Most of the problems in engineering, medical science, economics, environments, and so forth, have various uncertainties and vagueness. The existing models i.e. probability theory, fuzzy set theory etc., are insufficient for tackling such uncertainties and vagueness proficiently. Some sort of parameterization tool is lacking in this regards. In order to address such inadequacy, Molodtsov [19] initiated the concept of soft set theory as a mathematical tool for dealing with uncertainties and vagueness. This model is more effective and flexible as it is free from all such impediments. Maji et al. [15] developed gluing concept of fuzzy set with soft set i.e. fuzzy soft set and discussed its some essential properties and results. Many researchers $[16,20,6,8,9,26,12,13,24]$ extended the soft 
set theory and discussed its properties i.e. subset, absolute set, not set etc., operations i.e. complement, intersection, union, restricted union, restricted intersection, extended union, symmetric difference, AND, OR etc., and applied them in decision making to solve daily life problems. Akram et al. [1] investigated GDM methods based on hesitant N-soft sets with applications in decision-making. Ali et al. [7] discussed parameter reductions of bipolar fuzzy soft sets with their decision-making algorithms. Fatimah et al. [11] developed N-soft sets and discussed their decision making algorithms with applications. Maji et al. $[17,1]$ investigated intuitionistic fuzzy soft set with fundamentals and essential properties and operations. Alkhazaleh et al. [2] introduced the concept of soft expert set which is the combination of soft set theory and expert system. They characterized its basic operations i.e. complement, union, intersection, AND, OR etc., and successfully applied the concept in decision-making problems. Alkhazaleh et al. $[3,4,5]$ extended the work on soft expert set and developed the theories of soft multi sets, possibility fuzzy soft set and fuzzy parameterized interval-valued fuzzy soft set to adequate their already proposed structures for other scenarios. Convexity plays a vital role in optimization, pattern classification, image processing and classification as well as in operation research. In order to tackle all such problems and disciplines for uncertain, incomplete and vague information, Deli [10] presented the novel notions of convex and concave set under soft set and fuzzy soft set environments and discussed some important results based on set inclusion and set theoretic operations. Majeed [14] introduced the concept of convex hull and cone for soft sets to meet the demand of computational geometry with uncertain and vague information. Salih et al. [25] conceptualized strictly and strongly convexity cum concavity on soft sets. Rahman et al. [21, 22, 23] discussed the various properties of convexity and concavity under fuzzy soft set, soft set and hypersoft set (an extension of soft set) environments with some modified results.

1.1. Motivation. Convexity is an essential concept in optimization, recognition and classification of certain patterns, processing and decomposition of images, discrete event simulation, duality problems and many other related topics in operation research, mathematical economics, numerical analysis and other mathematical sciences. Deli [10] provided a mathematical tool to tackle all such problems under soft set environment. Soft expert set theory is the combination of soft set and expert set. It is a generalized one as it addresses the limitation of soft set for the consideration of experts opinions. In order to make the existing convexity-like literature in line with such kind of scenario, literature demands to carve out a conceptual framework for solving such kind of problems under more generalized version i.e. soft expert set. Therefore, in this study, an abstract and analytical approach is utilized to develop a basic framework of convexity and concavity on soft expert sets along with some important results motivated from the concept presented in [10]. Examples of convexity and concavity on soft expert sets are presented too.

1.2. Organization of Paper. The rest of the paper is organized as:

Section 2 : recalls some basic definitions and terms regarding soft set, soft expert set and convexity cum concavity on soft sets.

Section 3 : presents the novel definitions of convex soft expert set and concave soft expert set and some of their properties are discussed.

Section 4 : concludes the paper. 


\section{Preliminaries}

In this section, some basic definitions and terms regarding the main study are presented from the literature.

Definition 2.1. [19]

Let $P(Z)$ denote power set of $Z$ (universe of discourse) and $F$ be a collection of parameters defining $Z$. A soft set $M$ over $Z$ is defined by mapping

$$
h_{M}: F \rightarrow P(Z)
$$

Definition 2.2. [2]

Assume that $Y$ be a set of specialists (operators) and $\mathcal{O}$ be a set of conclusions, $T=$ $F \times Y \times \mathcal{O}$ with $S \subseteq T$ where $Z$ denotes the universe, $F$ a set of parameters.

A pair $(H, S)$ is known as a soft expert set over $Z$, where $H$ is a mapping given by

$$
H: S \rightarrow P(Z)
$$

For simplicity we assume in this paper, two-valued opinions only in set $\mathcal{O}$, that is, $\mathcal{O}=\{0$ = disagree, $1=$ agree $\}$.

Example 2.3. Suppose that an organization manufactured modern kinds of its brands and intends to proceed the assessment of certain specialists about concerning these items. Let $Z=\left\{v_{1}, v_{2}, v_{3}, v_{4}\right\}$ be a set of items, $F=\left\{a_{1}, a_{2}, a_{3}, a_{4}\right\}$ a set of choice parameters and let $S=\left\{a_{1}, a_{2}, a_{3}\right\}$ be a subset of $F$ where $a_{1}=$ simple to utilize, $a_{2}=$ nature, $a_{3}=$ modest and let $Y=\{s, t, u\}$ be a set of specialists. Assume that the organization has appropriated a survey to three specialists to settle on choices on the organization's items, and we get the accompanying:

$$
\begin{aligned}
& H_{1}=H\left(a_{1}, s, 1\right)=\left\{v_{1}, v_{2}, v_{4}\right\}, \\
& H_{3}=H\left(a_{1}, u, 1\right)=\left\{v_{3}, v_{4}\right\}, \\
& H_{5}=H\left(a_{2}, t, 1\right)=\left\{v_{1}, v_{3}\right\}, \\
& H_{7}=H\left(a_{3}, s, 1\right)=\left\{v_{3}, v_{4}\right\}, \\
& H_{9}=H\left(a_{3}, u, 1\right)=\left\{v_{4}\right\}, \\
& H_{11}=H\left(a_{1}, t, 0\right)=\left\{v_{2}, v_{3}\right\}, \\
& H_{13}=H\left(a_{2}, s, 0\right)=\left\{v_{1}, v_{2}, v_{3}\right\}, \\
& H_{15}=H\left(a_{2}, u, 0\right)=\left\{v_{3}\right\}, \\
& H_{17}=H\left(a_{3}, t, 0\right)=\left\{v_{3}, v_{4}\right\}, \\
& \text { We have soft expert set as follows }
\end{aligned}
$$$$
H_{2}=H\left(a_{1}, t, 1\right)=\left\{v_{1}, v_{4}\right\} \text {, }
$$$$
\begin{aligned}
& H_{4}=H\left(a_{2}, s, 1\right)=\left\{v_{4}\right\}, \\
& H_{6}=H\left(a_{2}, u, 1\right)=\left\{v_{1}, v_{2}, v_{4}\right\}, \\
& H_{8}=H\left(a_{3}, t, 1\right)=\left\{v_{1}, v_{2}\right\}, \\
& H_{10}=H\left(a_{1}, s, 0\right)=\left\{v_{3}\right\}, \\
& H_{12}=H\left(a_{1}, u, 0\right)=\left\{v_{1}, v_{2}\right\}, \\
& H_{14}=H\left(a_{2}, t, 0\right)=\left\{v_{2}, v_{4}\right\}, \\
& H_{16}=H\left(a_{3}, s, 0\right)=\left\{v_{1}, v_{2}\right\}, \\
& H_{18}=H\left(a_{3}, u, 0\right)=\left\{v_{1}, v_{2}, v_{3}\right\},
\end{aligned}
$$ 


$$
(H, S)=\left\{\begin{array}{l}
H_{1}=H\left(a_{1}, s, 1\right)=\left\{v_{1}, v_{2}, v_{4}\right\}, H_{2}=H\left(a_{1}, t, 1\right)=\left\{v_{1}, v_{4}\right\}, \\
H_{3}=H\left(a_{1}, u, 1\right)=\left\{v_{3}, v_{4}\right\}, H_{4}=H\left(a_{2}, s, 1\right)=\left\{v_{4}\right\}, \\
H_{5}=H\left(a_{2}, t, 1\right)=\left\{v_{1}, v_{3}\right\}, H_{6}=H\left(a_{2}, u, 1\right)=\left\{v_{1}, v_{2}, v_{4}\right\} \\
H_{7}=H\left(a_{3}, s, 1\right)=\left\{v_{3}, v_{4}\right\}, H_{8}=H\left(a_{3}, t, 1\right)=\left\{v_{1}, v_{2}\right\}, \\
H_{9}=H\left(a_{3}, u, 1\right)=\left\{v_{4}\right\}, H_{10}=H\left(a_{1}, s, 0\right)=\left\{v_{3}\right\}, \\
H_{1} 1=H\left(a_{1}, t, 0\right)=\left\{v_{2}, v_{3}\right\}, H_{12}=H\left(a_{1}, u, 0\right)=\left\{v_{1}, v_{2}\right\} \\
H_{1} 3=H\left(a_{2}, s, 0\right)=\left\{v_{1}, v_{2}, v_{3}\right\}, H_{14}=H\left(a_{2}, t, 0\right)=\left\{v_{2}, v_{4}\right\}, \\
H_{1} 5=H\left(a_{2}, u, 0\right)=\left\{v_{3}\right\}, H_{16}=H\left(a_{3}, s, 0\right)=\left\{v_{1}, v_{2}\right\}, \\
H_{1} 7=H\left(a_{3}, t, 0\right)=\left\{v_{3}, v_{4}\right\}, H_{18}=H\left(a_{3}, u, 0\right)=\left\{v_{1}, v_{2}, v_{3}\right\}
\end{array}\right\}
$$

See that from the above pattern the first specialist, $s$, "concurs" that the "simple to utilize" items are $v_{1}, v_{2}$, and $v_{4}$. The subsequent specialist $t$, "concurs" that the "simple to utilize" items are $v_{1}$ and $v_{4}$, and the third master, $u$, "concurs" that the "simple to utilize" items are $v_{3}$ and $v_{4}$. See here every one of specialists "concur" that item $v_{4}$ is "anything but simple to utilize."

Definition 2.4. [2]

A soft expert set $(H, S)$ is said to be soft expert subset of another soft expert set $(J, P)$, denoted by $(H, S) \subseteq(J, P)$ over $Z$, if

(i) $S \subseteq P$,

(ii) $\forall \alpha \in S, H(\alpha) \subseteq J(\alpha)$.

And $(J, P)$ is said to be a soft expert superset of $(H, S)$.

Definition 2.5. [2]

Let $F=\left\{\epsilon_{1}, \epsilon_{2}, \ldots, \epsilon_{n}\right\}$ be taken as a collection of parameters. The NOT set $\rceil F$ is characterized by \{\rceil$\left.\left.\left.\epsilon_{1},\right\rceil \epsilon_{2}, \ldots,\right\rceil \epsilon_{n}\right\}$ where $\rceil \epsilon_{i}=$ not $\epsilon_{i}, \forall i$.

Definition 2.6. [2]

The compliment of a soft expert set $(H, S)$ is meant by $(H, S)^{\prime}$ and is characterized by $\left.(H, S)^{\prime}=\left(H^{\prime},\right\rceil S\right)$ where $\left.H^{\prime}:\right\rceil S \rightarrow P(Z)$ is a mapping given by $\left.H^{\prime}(\xi)=Z-H(\rceil \xi\right)$ for every one of the $\xi \in\rceil S$.

Definition 2.7. [2]

The union of two soft expert sets $(H, S)$ and $(J, P)$ over $Z$ indicated by $(H, S) \cup(J, P)$, is the soft expert set $(K, L)$ where $L=S \cup P$, and $\forall \xi \in L$,

$$
K(\xi)=\left\{\begin{array}{cl}
H(\xi) & ; \xi \in S-P \\
J(\xi) & ; \xi \in P-S \\
H(\xi) \cup J(\xi) & ; \xi \in S \cap P
\end{array}\right.
$$

Definition 2.8. [2]

The intersection of two soft expert sets $(H, S)$ and $(J, P)$ over $Z$ indicated by $(H, S) \cap$ $(J, P)$ is the soft expert set $(K, L)$ where $L=S \cap P$ and $\forall \xi \in L$,

$$
K(\xi)=\left\{\begin{array}{cc}
H(\xi) & ; \xi \in S-P \\
J(\xi) & ; \xi \in P-S \\
H(\xi) \cap J(\xi) & ; \xi \in S \cap P
\end{array}\right.
$$


Definition 2.9. [10]

The soft set $S$ on $F$ is called a convex soft set if

$$
h_{S}\left(\xi \epsilon_{1}+(1-\xi) \epsilon_{2}\right) \supseteq h_{S}\left(\epsilon_{1}\right) \cap h_{S}\left(\epsilon_{2}\right)
$$

for each $\epsilon_{1}, \epsilon_{2} \in F$ and $\xi \in \Gamma^{\bullet}=[0,1]$.

Definition 2.10. [10]

The soft set $S$ on $F$ is called a concave soft set if

$$
h_{S}\left(\xi \epsilon_{1}+(1-\xi) \epsilon_{2}\right) \subseteq h_{S}\left(\epsilon_{1}\right) \cup h_{S}\left(\epsilon_{2}\right)
$$

for each $\epsilon_{1}, \epsilon_{2} \in F$ and $\xi \in \Gamma^{\bullet}=[0,1]$.

\section{Convex and Concave Soft Expert Sets}

In this portion, we describe the definition of convex soft expert set and concave soft expert set. In this article $F$ will denote the n-dimensional Euclidean space $R^{n}$ and $Z$ describes the arbitrary set.

Definition 3.1. Convex soft expert set

The soft expert set over $F$ is called a convex soft expert set if

$$
h_{S E S}\left(\xi \epsilon_{1}+(1-\xi) \epsilon_{2}\right) \supseteq h_{S E S}\left(\epsilon_{1}\right) \cap h_{S E S}\left(\epsilon_{2}\right)
$$

for each $\epsilon_{1}, \epsilon_{2} \in F$ and $\xi \in \Gamma^{\bullet}=[0,1]$.

Example 3.2. Referring to example (2.3) with $S=\left\{a_{1}, a_{2}, a_{3}\right\}=\{1,2,3\}$, and $Y=$ $\{s, t, u\}=\{1,2,3\} H_{1}=h_{S E S}(1,1,1)=\left\{v_{1}, v_{2}, v_{4}\right\}, H_{2}=h_{S E S}(1,2,1)=\left\{v_{3}, v_{4}\right\}$ Take $\xi=0.6$, and $\epsilon_{1}=(1,1,1), \epsilon_{2}=(1,2,1)$ then

$h_{S E S}\left(\xi \epsilon_{1}+(1-\xi) \epsilon_{2}\right)=h_{S E S}(0.6(1,1,1)+(1-0.6)(1,2,1))$

$=h_{S E S}((0.6,0.6,0.6)+(0.4,0.8,0.4))=h_{S E S}(1,1.4,1)=h_{S E S}(1,1,1)=\left\{v_{1}, v_{2}, v_{4}\right\}$ and $h_{S E S}\left(\epsilon_{1}\right) \cap h_{S E S}\left(\epsilon_{2}\right)=h_{S E S}(1,1,1) \cap h_{S E S}(1,2,1)=\left\{v_{1}, v_{2}, v_{4}\right\} \cap\left\{v_{3}, v_{4}\right\}=$ $\left\{v_{4}\right\}$

It is clear that

$$
h_{S E S}\left(\xi \epsilon_{1}+(1-\xi) \epsilon_{2}\right) \supseteq h_{S E S}\left(\epsilon_{1}\right) \cap h_{S E S}\left(\epsilon_{2}\right)
$$

Definition 3.3. Concave soft expert set

The soft expert set on $F$ is said to be a concave soft expert set if

$$
h_{S E S}\left(\xi \epsilon_{1}+(1-\xi) \epsilon_{2}\right) \subseteq h_{S E S}\left(\epsilon_{1}\right) \cup h_{S E S}\left(\epsilon_{2}\right)
$$

for each $\epsilon_{1}, \epsilon_{2} \in F$ and $\xi \in \Gamma^{\bullet}$.

Example 3.4. Considering the data from example (2.3), we have

$h_{S E S}\left(\epsilon_{1}\right) \cup h_{S E S}\left(\epsilon_{2}\right)=h_{S E S}(1,1,1) \cup h_{S E S}(1,2,1)=\left\{v_{1}, v_{2}, v_{4}\right\} \cup\left\{v_{3}, v_{4}\right\}=\left\{v_{1}, v_{2}, v_{3}, v_{4}\right\}$ and $h_{S E S}\left(\xi \epsilon_{1}+(1-\xi) \epsilon_{2}\right)=\left\{v_{1}, v_{2}, v_{4}\right\}$

It is clear that

$$
h_{S E S}\left(\xi \epsilon_{1}+(1-\xi) \epsilon_{2}\right) \subseteq h_{S E S}\left(\epsilon_{1}\right) \cup h_{S E S}\left(\epsilon_{2}\right)
$$


Definition 3.5. Suppose $R$ be a soft expert set (SES) over $Z$ and $\rho$ be subset of $Z$. Subsequently $\rho$-inclusion of $R$ is defined as

$$
R^{\rho}=\left\{\epsilon \in F: g_{R}(\epsilon) \supseteq \rho\right\}
$$

Theorem 3.6. $G_{1} \cap G_{2}$ is a convex soft expert set while $G_{1}$ and $G_{2}$ are convex SESs.

Proof. Assume $\exists \epsilon_{1}, \epsilon_{2} \in F$ and $\xi \in[0,1]$, and $G_{3}=G_{1} \cap G_{2}$.

$$
\begin{aligned}
& h_{S E S\left(G_{3}\right)}\left(\xi \epsilon_{1}+(1-\xi) \epsilon_{2}\right)= \\
& h_{S E S\left(G_{1}\right)}\left(\xi \epsilon_{1}+(1-\xi) \epsilon_{2}\right) \cap h_{S E S\left(G_{2}\right)}\left(\xi \epsilon_{1}+(1-\xi) \epsilon_{2}\right)
\end{aligned}
$$

$\because G_{1}$ and $G_{2}$ are convex SESs, so

$$
\begin{aligned}
& h_{S E S\left(G_{1}\right)}\left(\xi \epsilon_{1}+(1-\xi) \epsilon_{2}\right) \\
& h_{S E S\left(G_{2}\right)}\left(\xi \epsilon_{1}+(1-\xi) \epsilon_{2}\right) \supseteq h_{S E S\left(G_{1}\right)}\left(\epsilon_{1}\right) \cap h_{S E S\left(G_{2}\right)}\left(\epsilon_{1}\right) \cap h_{S E S\left(G_{2}\right)}\left(\epsilon_{2}\right) \\
& \therefore \\
& h_{S E S\left(G_{3}\right)}\left(\xi \epsilon_{1}+(1-\xi) \epsilon_{2}\right) \supseteq\left(h_{S E S\left(G_{1}\right)}\left(\epsilon_{1}\right) \cap h_{S E S\left(G_{1}\right)}\left(\epsilon_{2}\right)\right) \cap\left(h_{S E S\left(G_{2}\right)}\left(\epsilon_{1}\right) \cap h_{S E S\left(G_{2}\right)}\left(\epsilon_{2}\right)\right) \\
& . \\
& h_{S E S\left(G_{3}\right)}\left(\xi \epsilon_{1}+(1-\xi) \epsilon_{2}\right) \supseteq h_{S E S\left(G_{3}\right)}\left(\epsilon_{1}\right) \cap h_{S E S\left(G_{3}\right)}\left(\epsilon_{2}\right)
\end{aligned}
$$

Remark 3.7. If $\left\{G_{k}: k \in\{1,2,3, \ldots, n\}\right\}$ is finite collection of convex SESs, then the $\bigcap_{k=1}^{n} G_{k}$ is a convex $S E S$.

Theorem 3.8. $D$ is convex soft expert set( $(S E S)$ on $F$ iff for each $\eta \in[0,1]$ and $\rho \in P(Z)$, $D^{\rho}$ is convex SES on F.

Proof. : Suppose that $D$ is convex SES. If $\epsilon_{1}, \epsilon_{2} \in F$ and $\rho \in P(Z)$, then $h_{S E S(D)}\left(\epsilon_{1}\right) \supseteq$ $\rho$ and $h_{S E S(D)}\left(\epsilon_{2}\right) \supseteq \rho$. By the convexity of $D$ we have

$$
h_{S E S(D)}\left(\eta \epsilon_{1}+(1-\eta) \epsilon_{2}\right) \supseteq h_{S E S(D)}\left(\epsilon_{1}\right) \cap h_{S E S(D)}\left(\epsilon_{2}\right)
$$

so $D^{\rho}$ is convex $S E S$.

Conversely suppose that $D^{\rho}$ is convex SES for each $\eta \in[0,1]$. Then, for $\epsilon_{1}, \epsilon_{2} \in F, D^{\rho}$ is convex SES for $\rho=h_{S E S(D)}\left(\epsilon_{1}\right) \cap h_{S E S(D)}\left(\epsilon_{2}\right)$.

$\because h_{S E S(D)}\left(\epsilon_{1}\right) \supseteq \rho$ and $h_{S E S(D)}\left(\epsilon_{2}\right) \supseteq \rho$,

we have $\epsilon_{1} \in D^{\rho}$ and $\epsilon_{2} \in D^{\rho}$,

hence $\eta \epsilon_{1}+(1-\eta) \epsilon_{2} \in D^{\rho}$.

$\therefore h_{S E S(D)}\left(\eta \epsilon_{1}+(1-\eta) \epsilon_{2}\right) \supseteq h_{S E S(D)}\left(\epsilon_{1}\right) \cap h_{S E S(D)}\left(\epsilon_{2}\right)$,

which implies that $D$ is convex SES.

Remark 3.9. If $\left\{T_{k}: k \in\{1,2,3, \ldots\}\right\}$ is any collection of convex SESs, then $\bigcap_{k \in I} T_{k}$ is a convex SES.

Theorem 3.10. $T^{\prime}$ is concave SES while $T$ is convex SES. 
Proof. : Assume that $\exists \epsilon_{1}, \epsilon_{2} \in F, \xi \in \Gamma^{\bullet}$.

$\because T$ is convex SES,

$$
h_{S E S(T)}\left(\xi \epsilon_{1}+(1-\xi) \epsilon_{2}\right) \supseteq h_{S E S(T)}\left(\epsilon_{1}\right) \cap h_{S E S(T)}\left(\epsilon_{2}\right)
$$

or

$$
Z \backslash h_{S E S(T)}\left(\xi \epsilon_{1}+(1-\xi) \epsilon_{2}\right) \subseteq Z \backslash\left\{h_{S E S(T)}\left(\epsilon_{1}\right) \cap h_{S E S(T)}\left(\epsilon_{2}\right)\right\}
$$

If $h_{S E S(T)}\left(\epsilon_{1}\right) \supseteq h_{S E S(T)}\left(\epsilon_{2}\right)$ then we may write

$$
Z \backslash h_{S E S(T)}\left(\xi \epsilon_{1}+(1-\xi) \epsilon_{2}\right) \subseteq Z \backslash h_{S E S(T)}\left(\epsilon_{2}\right)
$$

If $h_{S E S(T)}\left(\epsilon_{1}\right) \subseteq h_{S E S(T)}\left(\epsilon_{2}\right)$ then we may write

$$
Z \backslash h_{S E S(T)}\left(\xi \epsilon_{1}+(1-\xi) \epsilon_{2}\right) \subseteq Z \backslash h_{S E S(T)}\left(\epsilon_{1}\right)
$$

From (1) and (2) equations, we have

$$
Z \backslash h_{S E S(T)}\left(\xi \epsilon_{1}+(1-\xi) \epsilon_{2}\right) \subseteq\left\{Z \backslash h_{S E S(T)}\left(\epsilon_{1}\right) \cup Z \backslash h_{S E S(T)}\left(\epsilon_{2}\right)\right\} .
$$

So, $T^{\prime}$ is concave SES.

Theorem 3.11. $G^{\prime}$ is convex SES while $G$ is concave SES.

Proof. : Assume that $\exists \epsilon_{1}, \epsilon_{2} \in F, \xi \in \Gamma^{\bullet}$.

$\because G$ is concave SES,

$$
h_{S E S(G)}\left(\xi \epsilon_{1}+(1-\xi) \epsilon_{2}\right) \subseteq h_{S E S(G)}\left(\epsilon_{1}\right) \cup h_{S E S(G)}\left(\epsilon_{2}\right)
$$

or

$$
Z \backslash h_{S E S(G)}\left(\xi \epsilon_{1}+(1-\xi \epsilon)_{2}\right) \supseteq Z \backslash\left\{h_{S E S(G)}\left(\epsilon_{1}\right) \cup h_{S E S(G)}\left(\epsilon_{2}\right)\right\}
$$

If $h_{S E S(G)}\left(\epsilon_{1}\right) \supseteq h_{S E S(G)}\left(\epsilon_{2}\right)$ then we may write

$$
Z \backslash h_{S E S(G)}\left(\xi \epsilon_{1}+(1-\xi) \epsilon_{2}\right) \supseteq Z \backslash h_{S E S(G)}\left(\epsilon_{1}\right)
$$

If $h_{S E S(G)}\left(\epsilon_{1}\right) \subseteq h_{S E S(G)}\left(\epsilon_{2}\right) \therefore$

$$
Z \backslash h_{S E S(G)}\left(\xi \epsilon_{1}+(1-\xi) \epsilon_{2}\right) \supseteq Z \backslash h_{S E S(G)}\left(\epsilon_{2}\right)
$$

From (4) and (5), we have

$$
Z \backslash h_{S E S(G)}\left(\xi \epsilon_{1}+(1-\xi) \epsilon_{2}\right) \supseteq\left\{Z \backslash h_{S E S(G)}\left(\epsilon_{1}\right) \cap Z \backslash h_{S E S(G)}\left(\epsilon_{2}\right)\right\}
$$

which shows that $G^{\prime}$ is convex SES.

Theorem 3.12. $N$ is concave SES on $F$ iff for each $\eta \in[0,1]$ and $\rho \in P(Z)$, $N^{\rho}$ is concave SES on $F$.

Proof. Suppose that $N$ is concave SES. If $\epsilon_{1}, \epsilon_{2} \in F$ and $\rho \in P(Z)$, then $h_{S E S(N)}\left(\epsilon_{1}\right) \supseteq$ $\rho$ and $h_{S E S(N)}\left(\epsilon_{2}\right) \supseteq \rho$. By the concavity of $N$, we have

$$
h_{S E S(N)}\left(\eta \epsilon_{1}+(1-\eta) \epsilon_{2}\right) \subseteq h_{S E S(N)}\left(\epsilon_{1}\right) \cup h_{S E S(N)}\left(\epsilon_{2}\right)
$$

hence $N^{\rho}$ is a concave SES.

Conversely suppose that $N^{\rho}$ is concave SES for each $\eta \in[0,1]$. Subsequently, for $\epsilon_{1}, \epsilon_{2} \in$ $F, N^{\rho}$ is concave SES for $\rho=h_{S E S(N)}\left(\epsilon_{1}\right) \cup h_{S E S(N)}\left(\epsilon_{2}\right)$.

$\therefore h_{S E S(N)}\left(\epsilon_{1}\right) \supseteq \rho$ and $h_{S E S(N)}\left(\epsilon_{2}\right) \supseteq \rho$, we have $\epsilon_{1} \in N^{\rho}$ and $\epsilon_{2} \in N^{\rho}$, hence $\eta \epsilon_{1}+(1-\eta) \epsilon_{2} \in N^{\rho} \therefore$ 
$h_{S E S(N)}\left(\eta \epsilon_{1}+(1-\eta) \epsilon_{2}\right) \subseteq h_{S E S(N)}\left(\epsilon_{1}\right) \cup h_{S E S(N)}\left(\epsilon_{2}\right)$, it is clear that $N$ is concave SES.

\section{CONCLUSION}

In this article, convexity and concavity is developed for soft expert set. Some of its essential properties i.e. complement, intersection and union are proved. Future work may include the

(1) Introduction of strictly and strongly convexity cum concavity on soft expert.

(2) Characterization of convex hull and convex cone on soft expert.

(3) Development of many other variants of convexity like $(m, n)$-convexity, $(m, n)$ convexity in 1st sense and 2nd sense, $\phi$-convexity, graded convexity, triangular convexity, harmonic convexity, concavo convexity etc. on soft expert set.

(4) The extension of this work for other structures like fuzzy soft set, intuitionistic fuzzy soft set, neutrosophic soft set, hypersoft set, fuzzy hypersoft set, intuitionistic fuzzy hypersoft set, neutrosophic hypersoft set etc.

It may also include the extension of this work by considering the modified versions of complement, intersection and union.

\section{REFERENCES}

[1] M. Akram, A. Adeel, J. C. R. Alcantud, Group decision-making methods based on hesitant $N$-soft sets, Expert Systems with Applications 115, (2019) 95-105.

[2] S. Alkhazaleh, A. R. Salleh, Soft Expert Sets, Advances in Decision Sciences 2011, (2011) 1-12, Article ID 757868. https://doi.org/10.1155/2011/757868.

[3] S. Alkhazaleh, A. R. Salleh, N. Hassan, Soft multi sets theory, Applied Mathematical Sciences 5, No.72 (2011) 3561-3573.

[4] S. Alkhazaleh, A. R. Salleh, N. Hassan, Possibility fuzzy soft set, Advances in Decision Sciences 2011 (2011) 1-18, Article ID 479756. https://doi.org/10.1155/2011/479756

[5] S. Alkhazaleh, A. R. Salleh, N. Hassan, Fuzzy parameterized interval-valued fuzzy soft set, Applied Mathematical Sciences, 5, No. 67 (2011) 3335-3346.

[6] M.I. Ali, F. Feng, X. Liu, W.K. Min, M. Sabir, On some new operations in soft set theory, Computers and Mathematics with Applications 57, (2009) 1547-1553.

[7] G. Ali, M. Akram, A. N. Koam, J. C. R. Alcantud, Parameter reductions of bipolar fuzzy soft sets with their decision-making algorithms, Symmetry, 11, No.8 (2019) 949.

[8] K.V. Babitha, J.J. Sunil, Soft set relations and functions, Computers and Mathematics with Applications 60 , (2010) 1840-1849.

[9] K.V. Babitha, J.J. Sunil, Transitive closure and ordering in soft set, Computers and Mathematics with Applications 61, (2011) 2235-2239.

[10] I. Deli, Convex and Concave Sets Based on Soft Sets and Fuzzy Soft Sets, Journal of New Theory 29, (2019) 101-110.

[11] F. Fatimah, D. Rosadi, R. F. Hakim, J. C. R. Alcantud, $N$-soft sets and their decision making algorithms, Soft Computing 22, No. 12 (2018) 3829-3842.

[12] X. Ge, S. Yang, Investigations on some operations of soft sets, World Academy of Science Engineering and Technology 75, (2011) 1113-1116.

[13] F. Li, Notes on soft set operations, ARPN Journal of systems and softwares 1, No.6 (2011) 205-208.

[14] S. N. Majeed, Some notions on convex soft sets, Annals of Fuzzy Mathematics and Informatics, 12, No.4 (2016) 517-526.

[15] P.K. Maji, R. Biswas, A.R. Roy, Fuzzy soft sets, Journal of Fuzzy Mathematics 9, No.3 (2001) 589-602.

[16] P.K. Maji, R. Biswas, A.R. Roy, Soft Set Theory, Computers and Mathematics with Applications 45, (2003) $555-562$. 
[17] P.K. Maji, R. Biswas, A.R. Roy, Intuitionistic fuzzy soft sets, The Journal of Fuzzy Mathematics, 9, No.3 (2001) 677-692.

[18] P. K. Maji, More on intuitionistic fuzzy soft sets, In International workshop on rough sets, fuzzy sets, data mining, and granular-soft computing (pp. 231-240), Springer, Berlin, Heidelberg, (2009).

[19] D. Molodtsov, Soft Set Theory - First Results, Computers and Mathematics with Applications 37, (1999) 19-31.

[20] D. Pei, D. Miao, From soft set to information system, In international conference of granular computing, IEEE 2, (2005) 617-621.

[21] A. U. Rahman, M. Saeed, M. Arshad, M. Ihsan, M. R. Ahmad, ( $m, n)$-Convexity-cum-Concavity on Fuzzy Soft Set with Applications in First and Second Sense, Punjab University Journal of Mathematics, 53, No.1 (2021) 19-33.

[22] A. U. Rahman, M. Saeed, M. Ihsan, M. Arshad, S. Ayaz, A Conceptual Framework Of m-Convex And mConcave Sets Under Soft Set Environment With Properties, Transactions in Mathematical and Computational Sciences, 1, No.1 (2021) 5060.

[23] A. U. Rahman, M. Saeed, F. Smarandache, Convex and Concave Hypersoft Sets with Some Properties, Neutrosophic Sets and Systems, 38, (2020) 497-508.

[24] M. Riaz, N. Şagman, N. Wali, A. Mushtaq, Certain properties of soft multi-set topology with applications in multi-criteria decision making. Decision Making, Applications in Management and Engineering, 3, No.2 (2020) 70-96.

[25] H. M. Salih, Pishtiwan O. Sabir, Some New Properties of Convex and Concave Soft Sets, Diyala Journal for Pure Sciences 15, No.1 (2019) 84-94.

[26] A. Sezgin, A.O. Atagün, On operations of soft sets, Computers and Mathematics with Applications 61, No.5 (2011) 1457-1467. 